\title{
Chemistry in Protoplanetary Disks
}

\author{
Yuri Aikawa \\ Department of Earth and Planetary Sciences, Kobe University, Kobe \\ 657-8501, Japan
}

\begin{abstract}
Molecular evolution is calculated in migrating fluid parcels in a protoplanetary disk considering gas-phase and grain-surface reactions. Radial distribution of molecules is obtained, which depends on ionization rate, temperature and/or desorption rate in the disk. If the temperature is high enough $(>20$ $\mathrm{K}$ ) or non-thermal desorption is efficient enough to sublimate dominant species such as $\mathrm{CO}$ in the comet forming regions, the desorbed species are transformed to less volatile species and again incorporated into ice mantles. Chemistry in these outer disks is similar to that in molecular clouds; it produces both oxidized and reduced species with a high $\mathrm{D} / \mathrm{H}$ ratio.
\end{abstract}

\section{Introduction}

Chemistry in the protoplanetary disk is a key to understand the evolution from interstellar matter to planetary matter. Spectroscopic observation of disks detected several molecules and determined their relative abundances in the gas phase (Dutrey et al. 1997; van Zadelhoff et al. 2001; Aikawa et al. 2003; Qi et al. 2003) and ice mantles (Thi et al. 2002). But the current observations are sensitive only to the regions outside $100 \mathrm{AU}$ owing to the poor angular resolution. Comets are unique probes in planet-forming regions until we get new facilities such as ALMA. In this contribution I solve the chemical reaction network in the disk, and obtain ice composition in the comet-forming region at $R=5-20 \mathrm{AU}$. The model is basically the same as Aikawa \& Herbst (1999), but the current work adopts updated disk model and includes grain-surface reactions.

\section{2. $\quad$ Model}

A steady accretion disk model by D'Alessio et al. (1999) is adopted. They solve energy transfer (including the irradiation from the central star and gravitational energy released by accretion) to determine vertical structure of the disk at each radius. In the midplane of comet-forming region, the temperature is $\sim 30-60$ $\mathrm{K}$ and gas density is $\sim 10^{11}-10^{12} \mathrm{~cm}^{-3}$.

Chemical reaction network is solved as an initial value problem. The initial composition of gas and ice is given by calculating contraction of a molecular cloud core. Then we follow migrating fluid parcels in the disk towards the central star. The network includes gas-phase reactions, gas-dust interaction (adsorption and desorption), and modified-rate grain-surface reactions (Stantcheva et al. 2001). Gas-phase reaction includes cosmic ray ionization. Since the attenuation length of the cosmic-ray ionization $\left(96 \mathrm{~g} \mathrm{~cm}^{-2}\right)$ is larger than the column density to 
the midplane at $R=5 \mathrm{AU}$, the interstellar ionization rate $\sim 10^{-17} \mathrm{~s}^{-1}$ would be available in the disk. If the cosmic ray is scattered by magnetic fields of the central star, decay of ${ }^{26} \mathrm{Al}$ could be the main ionization source with the ionization rate of $6 \times 10^{-19} \mathrm{~s}^{-1}$ (Umebayashi \& Nakano 1981). The ionization rate by $\mathrm{X}$ rays from the central star is $>10^{-17} \mathrm{~s}^{-1}$ within the surface layer of $\leq 10^{24}$ $\mathrm{cm}^{-2}$ at $R=5 \mathrm{AU}$ (Igea \& Glassgold 1999). If ions and radicals produced in the upper layers can diffuse into the midplane, column density at $R=5 \mathrm{AU}$ (column-density averaged) ionization rate is $\sim 10^{-18} \mathrm{~s}^{-1}$. I investigate models with ionization rates of $10^{-18} \mathrm{~s}^{-1}$ and $10^{-17} \mathrm{~s}^{-1}$.

\section{Results}

\subsection{Temporal Evolution and Radial Distribution}

Fluid parcels of interstellar matter fall onto the disk at $R \sim 250 \mathrm{AU}$, and migrate to $R=5-20 \mathrm{AU}$ in $3 \times 10^{6} \mathrm{yr}$. Molecular abundances vary with time, and newly formed ice accretes onto grains, which are already covered by interstellar ice. Although the density is high, the chemistry in these outer radii is qualitatively similar to cloud chemistry. Figure 1 (a) shows radial distribution of molecules at $3 \mathrm{Myr}$, which corresponds to a typical life time of classical T Tauri stars. At $R \geq 15 A U$ ice composition is almost constant, which is determined in the stage of molecular cloud and migration in the outer radius, and is basically similar to interstellar ice. At $R \leq 15 \mathrm{AU}$ radial variation is more significant. For example, $\mathrm{H}_{2} \mathrm{~S}$ ice is transformed to $\mathrm{SO}$ ice after sublimation. $\mathrm{HCN}$ ice is more abundant in the inner radius. Just like hot core chemistry, the evaporation of simple species such as $\mathrm{CO}$ and $\mathrm{H}_{2} \mathrm{~S}$ triggers the new gas-phase reactions; the larger and less volatile species are formed and adsorbed onto ice mantle again.

\subsection{If the Gas is Initially Hot}

causes diffusion in the disk, and thermally processed material in inner hot regions might migrate to outer regions. Such diffusion or shock heating in the comet-forming regions is suggested from the existence of crystalline silicate in the disks and comets. Here I assume that the matter is initially processed at high temperature and left in the cold comet-forming region. Considering the equilibrium at high temperature, initial composition is assumed to be $\mathrm{CO}, \mathrm{N}_{2}$ and water. At $t=0$, the matter is put at the radius of $3-25 \mathrm{AU}$. Molecules observed in comets - such as hydrocarbons, $\mathrm{NH}_{3}, \mathrm{CO}_{2}$ and deuterated water - become abundant within a time scale of $10^{6}$ yr. Figure 1 (b) shows radial distribution of molecules at $3 \mathrm{Myr}$. The abundance varies with radius according to the temperature gradient in the disk.

\subsection{Implications for Comets}

If chemistry is inactive in comet forming regions, variation of comet composition is caused only by evaporation, and comets formed in outer radius have larger abundance of all molecular species. On the other hand, if chemistry is active in the comet-formic regions, as shown in this work, some species become more abundant in comets formed in inner regions. A'Hearn et al. (1995) found that carbon chain species are depleted in Jupiter-Family comets, dynamical origin of 

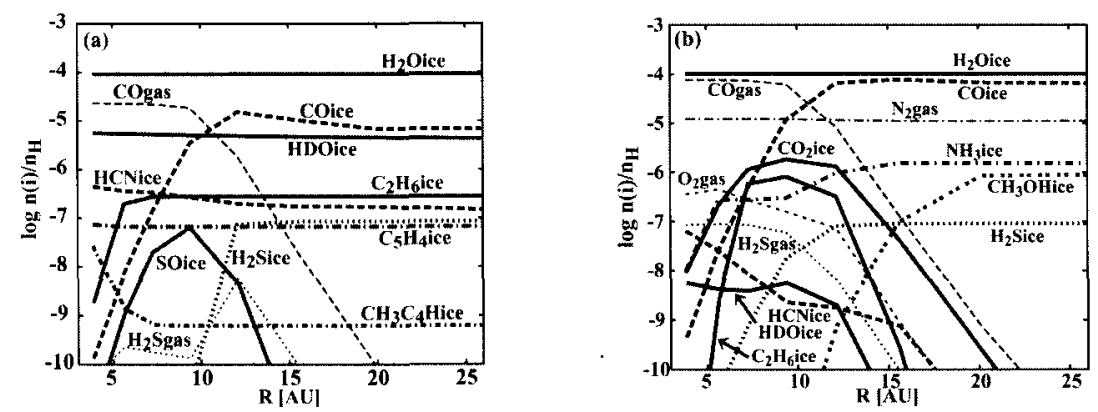

Figure 1. (a) Radial distribution of molecules in a steady accretion disk model at 3 Myr. The ionization rate is assumed to be $1.3 \times 10^{-17} \mathrm{~s}^{-1}$. (b) Radial distribution of molecules at $3 \mathrm{Myr}$. Initial composition is $\mathrm{CO}$ gas, $\mathrm{N}_{2}$ gas, and $\mathrm{H}_{2} \mathrm{O}$ ice. Ionization rate is assumed to be $1.3 \times 10^{-18} \mathrm{~s}^{-1}$.

which are in outer radius than that of the Halley-type comets. It may indicate the second case is appropriate for the Solar Nebula.

Acknowledgments. Y. A. is supported by a Grant-in-Aid for Scientific Research (14740130) and "The 21st Century COE Program of Origin and Evolution of Planetary Systems" of Ministry of Education, Culture, Sports, Science and Technology (MEXT) of Japan.

\section{References}

A'Hearn, M., Millis, R. L., Schleicher, D. G., Osip, D. J., \& Birch, P. V. 1995, Icarus, 118,223

Aikawa, Y., \& Herbst, E. 1999, ApJ, 526, 314

Aikawa, Y., Momose, M., Thi, W. F., van Zadelhoff, G. J., Qi, C., Blake, G. A., \& van Dishoeck, E. F. 2003, PASJ, 55, 11

D'Alessio, P., Calvet, N., Hartmenn, L., Lizano, S., \& Canto, J. 1999, ApJ, 527, 893

Dutrey, A., Guilloteau, S., \& Guelin, M. 1997, A\&A, 317, L55

Igea, J., \& Glassgold, A. E. 1999, ApJ, 518, 848

Qi, C., Kessler, J. E., Koerner, D. W., Blake, G. A., \& Sargent, A. I. 2003, ApJ, 597, 986

Stantcheva, T., Caselli, P., \& Herbst, E. 2001, A\&A, 375, 673

Thi, W. F., Pontoppidan, K. M., van Dishoeck, E. F., Dartois, E., d'Hendecourt, L. 2002, A\&A, 394, 27

Umebayashi, T., \& Nakano, T. 1981, PASJ, 33, 617

van Zadelhoff, G. J., van Dishoeck, E. F., Thi, W. F., Blake, G. A. 2001, A\&A, 377, 566 